\title{
Electroacupuncture promotes axonal regeneration in rats with focal cerebral ischemia through the downregulation of Nogo-A/NgR/RhoA/ROCK signaling
}

\author{
SAIE HUANG $^{1 *}$, DANXIA HUANG ${ }^{2 *}$, JIAPEI ZHAO ${ }^{3}$ and LIDIAN CHEN ${ }^{1}$ \\ ${ }^{1}$ Department of Neurological Rehabilitation, Fujian University of Traditional Chinese Medicine Subsidiary \\ Rehabilitation Hospital, Fuzhou, Fujian 350003; ${ }^{2}$ Department of Clinical Medicine, Quanzhou Medicine College, \\ Quzhou, Fujian 362000; ${ }^{3}$ Fujian Provincial Rehabilitation Industrial Institution, Department of Rehabilitation Medicine, \\ College of Rehabilitation Medicine, Fujian University of Traditional Chinese Medicine, Fuzhou, Fujian 350122, P.R. China
}

Received January 20, 2016; Accepted January 6, 2017

DOI: 10.3892/etm.2017.4621

\begin{abstract}
The purpose of the present study was to evaluate the effect of electroacupuncture (EA) on the axonal regeneration environment following cerebral ischemia injury and to investigate whether it was associated with Nogo-A/Nogo receptor $(\mathrm{NgR}) / \mathrm{RhoA} / \mathrm{Rho}$-associated protein kinase (ROCK) signaling. Using a rat model of focal cerebral ischemia, the effects of EA at the Quchi (LI11) and Zusanli (ST36) acupoints on axonal growth inhibitory protein and axonal growth factors were assessed and the underlying molecular mechanisms were investigated. It was found that EA at the Quchi and Zusanli acupoints significantly improved neurological deficit scores following ischemia $(\mathrm{P}<0.05)$, and reduced the cerebral infarct volume. Moreover, it was demonstrated that crucial signaling molecules in the Nogo-A signaling pathway were regulated by EA. These results suggest that EA provides a less inhibitory environment for axonal regeneration following cerebral ischemia through inhibition of Nogo-A/NgR/RhoA/ROCK signaling.
\end{abstract}

Correspondence to: Dr Lidian Chen, Department of Neurological Rehabilitation, Fujian University of Traditional Chinese Medicine Subsidiary Rehabilitation Hospital, 13 Hudong Road, Fuzhou, Fujian 350003, P.R. China

E-mail: sunshine11shine@163.com

*Contributed equally

Abbreviations: GAP-43, growth-associated protein 43; EA, electroacupuncture; IHC, immunohistochemical; MCAO, middle cerebral artery occlusion; CCA, common carotid artery; ECA, external carotid artery; ICA, internal carotid artery; DAB, diaminobenzidine; SC, sham operation control; IC, ischemia control

Key words: electroacupuncture, cerebral ischemia, Nogo-A, axonal regeneration, stroke

\section{Introduction}

Stroke is a major cause of mortality and permanent disability in adults worldwide, particularly in low- and middle-income countries $(1,2)$. Approximately $87 \%$ of stroke cases are due to ischemia (3). In the acute stages of the disease, neurons in the ischemic lesion rapidly die and other neuronal populations in the ischemic penumbra are vulnerable to secondary injury (4). Despite improvements in interventional techniques and pharmacological agents, including thrombolytic therapy, only a minority of stroke patients receive thrombolytic agents due to their side effects and narrow time window of administration (5). It is therefore critical to develop safe and effective treatments with a longer treatment time window.

Electroacupuncture (EA) was reported to have beneficial effects in stroke patients (6,7), and the Quchi (LI11) and Zusanli (ST36) acupoints are the most commonly used acupoints to treat stroke in the clinic in China. Preliminary studies by our group have demonstrated that EA on these two acupoints had a significant neuroprotective effect via the stimulation of cerebral cell proliferation (8-10). Neuroprotection and neural regeneration are two major therapeutic strategies to treat ischemic stroke (4). Previous studies have demonstrated that axonal regeneration has a central role in neural plasticity and may offer novel treatment approaches for stroke $(11,12)$. However, whether EA mediates axonal regeneration of injured neurons in rats and the possible underlying mechanism remains to be elucidated.

Nogo-A is a well-known myelin-associated axonal growth inhibitory protein, which has been shown to inhibit migration and spreading of nerve cells and has important roles in blocking axonal regeneration and reconnection following stroke $(13,14)$. Nogo-A inhibits axonal outgrowth by binding to Nogo receptor $(\mathrm{NgR})$ via its functional component to activate RhoA and its effector Rho-associated protein kinase (ROCK), leading to axonal repulsion and growth cone collapse (15). Studies have indicated that anti-Nogo-A therapy improves neurological deficits and enhances neuronal plasticity, suggesting that therapeutics targeting Nogo-A hold promise for regenerative treatment following stroke $(16,17)$. Therefore, 
the purpose of the present study was to test the hypothesis that the downregulation of Nogo-A contributes to the axonal regeneration effects of EA at the Zusanli and Quchi acupoints, evaluate the therapeutic efficacy of EA against ischemic stroke and investigate whether its effect is associated with downregulation of the Nogo-A signaling pathway.

\section{Materials and methods}

Materials and reagents. TRIzol reagent was obtained from Invitrogen (Thermo Fisher Scientific, Waltham, MA, USA). Nogo-A (no. ab62024), NgR (no. ab26291) and RhoA (no. ab54835) antibodies were obtained from Abcam (Cambridge, MA, USA). ROCK, growth-associated protein 43 (GAP-43, no. 5307), $\beta$-actin (no. 3700) antibodies and anti-rabbit immunoglobulin ( $\mathrm{Ig}) \mathrm{G}(\mathrm{H}+\mathrm{L})$, biotinylated secondary antibodies (no. 14708) were purchased from Cell Signaling Technology, Inc.(Beverly, MA, USA). Mouse proliferating cell nuclear antigen immunohistochemical (IHC) kits were purchased from Beijing Golden Bridge Biotechnology Co., Ltd. (Beijing, China). All other reagents used, unless otherwise stated, were obtained from Sigma-Aldrich (Merck KGaA, Darmstadt, Germany).

Animals. The experimental animal protocol of the present study was approved by the Ethics Committee of Fujian University of Traditional Chinese Medicine (Fuzhou, China; no. 2014028). All experiments were performed in strict accordance with the Guide for the Care and Use of Laboratory Animals published by the US. National Institutes of Health (18). A total of 108 male Sprague Dawley rats (weighing 220-250 g and aged 2.21 \pm 0.15 months) were purchased from Shanghai SLAC Laboratory Animal Co., Ltd. (Shanghai, China) and kept in a $23 \pm 2{ }^{\circ} \mathrm{C}$ temperature- and $50 \pm 5 \%$ humidity-controlled room under a 12-h light/dark cycle. Rats had ad libitum access to food and water.

Establishment of the focal cerebral ischemia rat model and animal grouping. Rats $(\mathrm{n}=108)$ were randomly divided into three groups prior to middle cerebral artery occlusion (MCAO) surgery: The sham operation control group (SC; $\mathrm{n}=36$ ), the ischemia control group (IC; $n=36$ ) and the EA group ( $\mathrm{EA} ; \mathrm{n}=36)$. To establish the focal cerebral ischemia model, rats were subjected to MCAO, as described previously by Longa et al (19). Following fasting for $24 \mathrm{~h}$ with access to water, rats were anesthetized by intraperitoneal injection with $10 \%$ chloral hydrate $(300 \mathrm{mg} / \mathrm{kg}$, Beijing Golden Bridge Biotechnology Co., Ltd.). Throughout the surgical procedures, the rectal temperature of the rats was maintained at $37^{\circ} \mathrm{C}$. In brief, a midline cervical incision was performed to expose and isolate the left common carotid artery (CCA), the left external carotid artery (ECA) and the internal carotid artery (ICA). An embolus was advanced through the ICA to the MCA $(20 \pm 2 \mathrm{~mm})$ until a mild resistance was encountered, thereby occluding the origin of the MCA. Subsequently, the cervical incision was closed with a silk suture. Rats in the sham group were treated similarly, however no ligations or occlusions were performed. Animals in each group were randomly assigned to three subgroups to be sacrificed at days 3,7 or 14 after surgery.
EA stimulation. In the EA group, EA treatment was performed at the Zusanli (ST36) and Quchi (LI11) acupoints on the right paralyzed limb using an EA stimulator instrument (Model G6805; Shanghai Huayi Co., Shanghai, China) following recovery from MCAO surgery. Two 30 -gauge $(0.3 \mathrm{~mm}$ diameter) stainless acupuncture needles were inserted at a depth of 2-3 mm into the aforementioned acupuncture points. The stimulation parameters were a dense disperse wave of 1 and $20 \mathrm{~Hz}$ (adjusted to the muscle twitch threshold), $30 \mathrm{~min}$ each time, once a day. Treatment was started the day after the operation and continued until the animals were sacrificed.

Neurological assessment. Neurological deficits were evaluated to confirm successful establishment of the rat model or cerebral ischemia injury. The severity of neurological deficits in the model animals was assessed at 2 and $24 \mathrm{~h}$ after surgery according to the four-point scoring system developed by Longa et al (19) and Bederson et al (20): 0, no apparent deficits; 1 , failure to fully extend the right forepaw; 2 , circling to the right; 3 , falling to the right when walking; 4 , loss of ability to walk. MCAO rats with neurological deficit scores of 1-3 were used for the experiments. Neurological deficits in each group of rats were re-evaluated at each time-point (day 3,7 and 14 after surgery) prior to sacrifice.

Evaluation of infarct volumes. Following cerebral ischemia injury for $72 \mathrm{~h}$, the rats were sacrificed under deep anesthesia using $10 \%$ chloral hydrate $(300 \mathrm{mg} / \mathrm{kg})$ and transcardially perfused with $0.9 \% \mathrm{NaCl}$. The brains of all rats were frozen at $-80^{\circ} \mathrm{C}$ in a freezer for $20 \mathrm{~min}$ and were cut into five coronal slices at a thickness of $2 \mathrm{~mm} / \mathrm{section}$. The fresh slices were placed in a $2 \%$ solution of 2,3,5-triphenyl-tetrazolium chloride (TTC) in phosphate-buffered saline (PBS; Hyclone; GE Healthcare, Logan, UT, USA) at $37^{\circ} \mathrm{C}$ for $20 \mathrm{~min}$. The normal area of the brain was stained dark red based on intact mitochondrial function, whereas the infarct area remained unstained. Images of the stained slices were captured using a high-resolution digital camera (Canon SX20; Canon, Inc., Tokyo, Japan). A computerized image analysis system (Motic Med 6.0 System; Motic, Hong Kong, China) was used for quantification of the percentage of the total infarcted brain volume.

Immunohistochemistry (IHC). The rats in each group $(\mathrm{n}=6)$ were humanely sacrificed at 3,7 and 14 days after MCAO surgery. The tissue was subjected to routine paraffin embedding, sectioning at $5 \mu \mathrm{m}$ thickness and antigen retrieval, followed by rinsing with 0.01 M PBS. Nogo-A, ROCK and GAP-43 levels were analyzed using an IHC assay kit (DAB kit-0017; MaixinBiotech, Co. Ltd., Fujian, China) according to the manufacturer's instructions. To block non-specific protein activity, the sections were incubated in $3 \%$ hydrogen peroxide and $10 \%$ normal goat serum (DAB kit-0017; MaixinBiotech, Co. Ltd., Fujian, China) for $10 \mathrm{~min}$ at $37^{\circ} \mathrm{C}$. Subsequently, the sections were incubated overnight at $4^{\circ} \mathrm{C}$ with primary antibodies against Nogo-A (1:100 dilution), ROCK (1:100 dilution) and GAP-43 (1:200 dilution), followed by incubation with secondary biotinylated rabbit anti-mouse antibodies (1:100 dilution; DAB kit-0017; MaixinBiotech, Co. Ltd., Fujian, China). Following incubation with diaminobenzidine for $1 \mathrm{~min}$, sections were washed with distilled water and dehydrated in a graded series of alcohol, cleared in xylene 
Table I. Neurological deficit score of rats at different time-points following injury.

\begin{tabular}{lccccc}
\hline Group & $2 \mathrm{~h}$ & $24 \mathrm{~h}$ & $3 \mathrm{~d}$ & $7 \mathrm{~d}$ & $14 \mathrm{~d}$ \\
\hline SC & 0 & 0 & 0 & 0 & 0 \\
IC & $2.25 \pm 0.62$ & $2.17 \pm 0.39$ & $2.08 \pm 0.52$ & $1.92 \pm 0.52$ & $1.83 \pm 0.58$ \\
EA & $2.33 \pm 0.65$ & $2.17 \pm 0.58$ & $1.67 \pm 0.49^{\mathrm{a}}$ & $1.50 \pm 0.52^{\mathrm{a}}$ & $1.33 \pm 0.49^{\mathrm{b}}$ \\
\hline
\end{tabular}

${ }^{\mathrm{a}} \mathrm{P}<0.05$ vs. the IC group; ${ }^{\mathrm{b}}<0.001$ vs. the IC group. Values are expressed as the mean \pm standard deviation from 12 individual rats in each group. SC, sham operation control; IC, ischemic control; EA, electroacupuncture; h, hours; d, days.

and mounted using xylene-based mounting medium. Images were captured on a microscope (Leica Microsystems, Wetzlar, Germany) and cells with positive staining were counted in four randomly selected microscopic fields at x400 magnification. The positive expression rate was determined as the ratio of cells with brown staining and Image-Pro Plus 6.0 (Media Cybernetics, Inc., Rockville, MD, US) was used to count the number of positive cells.

Western blot analysis. The rats were sacrificed to obtain the left peri-infarct cerebral cortex from each group at each time-point (3, 7 and 14 days post-surgery; $n=6$ per group). Tissues were homogenized in RIPA Lysis Buffer (No. P0013, Beyotime Institute of Biotechnology, Haimen, China) and centrifuged at $12,000 \times \mathrm{g}$ for $15 \mathrm{~min}$ at $4^{\circ} \mathrm{C}$. The supernatants were collected and frozen at $-80^{\circ} \mathrm{C}$ prior to immunoblotting. The protein concentration of each homogenate was determined using a Bicinchoninic Acid Protein assay kit (No. CW0014, Beijing Comwin Biotech Co., Ltd., Beijing, China). A total of $50 \mu \mathrm{g}$ protein per lane was separated by $10 \%$ SDS-PAGE. Subsequently, blots were transferred onto polyvinylidene difluoride membranes (Shanghai Bioscience Co., Shanghai, China) in a Tris-glycine transfer buffer and were blocked for $2 \mathrm{~h}$ with $5 \%$ non-fat dry milk at room temperature. Membranes were incubated with primary antibodies against Nogo-A (1:1,000 dilution), NgR (1:10,00 dilution), RhoA (1:1,000 dilution), ROCK (1:1,000 dilution), GAP-43 (1:5,000 dilution) and $\beta$-actin (1:5,000 dilution) overnight at $4^{\circ} \mathrm{C}$, followed by incubation with the anti-rabbit $\operatorname{IgG}(\mathrm{H}+\mathrm{L})$, biotinylated secondary antibodies (1:5,000 dilution) for $1 \mathrm{~h}$ at $37^{\circ} \mathrm{C}$. The bands were visualized with the ECL Pico Western Blotting Substrate kit (Beijing Golden Bridge Biotechnology Co., Beijing, China), images were captured using a Bio-Rad ChemiDoc XRS+ (Bio-Rad Laboratories, Hercules, CA, USA) and densitometric quantification was performed using Quantity OneV4.62 (Bio-Rad Laboratories). The ratio of gray scale values of the target protein to the internal control was used to measure the relative amount of Raldh1 and Raldh2.

RNA extraction and reverse-transcription polymerase chain reaction $(R T-P C R)$. Rats were sacrificed to obtain the left peri-infarct cerebral cortex from each group each time-point (3, 7 and 14 days; $n=6$ per group). Total RNA was isolated with the TRIzol reagent according to the manufacturer's instructions. Primed RNA $(1 \mu \mathrm{g})$ was reverse-transcribed with RevertAid ${ }^{\mathrm{TM}}$ First Strand cDNA Synthesis kit (5X Mix included Buffer, dNTP, HiScript ${ }^{\circledR}$ Reverse Transcriptase and RNase inhibitor; Fermentas; Thermo Fisher Scientific Inc.) according to the manufacturer's instructions. The reaction conditions for reverse-transcription were $50^{\circ} \mathrm{C}$ for $15 \mathrm{~min}$, followed by $85^{\circ} \mathrm{C}$ for $2 \mathrm{~min}$. cDNA was then amplified by PCR to determine the amount of Nogo-A, NgR, RhoA, ROCK and GAP-43 mRNA. $\beta$-actin was selected as a housekeeping gene. Primers were purchased from Shanghai BioSun Sci\&Tech Co., Ltd. (Shanghai, China). Primer sequences were as follows: Nogo-A forward, 5'-AGGGAT GTGCTGGCTGCTAG-3' and reverse, 5'-GGTGCTTTC GGTTGCTGAGG-3'; NgR, forward, 5'-GGGCAACCTCAC GCATCTCT-3' and reverse, 5'-TCATGAGTCGGCCAAGGT CC-3'; RhoA forward, 5'-ACCAGTTCCCAGAGGTTTAT-3' and reverse, 5'-TTTGGTCTTTGCTGAACACT-3', ROCK forward, 5'-GATCCCCTGCAAAGTTTATT-3' and reverse, 5'-AGCTTTTCCAAAAATGCAAA-3'; GAP-43 forward, 5'-TGCTGTGCTGTATGAGAAGAACC-3' and reverse, 5'-GGCAACGTGGAAAGCCGTTT3'; $\beta$-actin forward, 5'-ACTGGCATTGTGATGGACTC-3' and reverse, 5'-CAG CACTGTGTTGGCATAGA-3'. Following the addition of $2 \mathrm{x}$ AceTaq $^{\circledR}$ Master Mix (no. P411, Vazyme biotech Co., Ltd.), which includes Buffer, dNTP and AceTaq ${ }^{\circledR}$ DNA Polymerase, a MasterCycler nexus PCR (No. 5331, Eppendorf Ltd., Hamburg, Germany) was used for the PCR reaction. The thermocycling conditions for real-time PCR were $95^{\circ} \mathrm{C}$ for $5 \mathrm{~min}$, followed by 35 cycles of $95^{\circ} \mathrm{C}$ for $30 \mathrm{sec}$, $55^{\circ} \mathrm{C}$ for $30 \mathrm{sec}$ and $72^{\circ} \mathrm{C}$ for $60 \mathrm{sec}$. Following 35 cycles, elongation was performed at $72^{\circ} \mathrm{C}$ for $7 \mathrm{~min}$. PCR products were analyzed by gel electrophoresis (1.5\% agarose) and a Gel Documentation system (Model Gel Doc 2000; Bio-Rad Laboratories, Inc.). Quantity One (Bio-Rad Laboratories) was used for quantification.

Statistical analysis. SPSS 18.0 for Windows (SPSS Inc., Chicago, IL, USA) was used for statistical analysis. Values are expressed as the mean \pm standard deviation. Differences between the two groups were compared using the independent-samples Student's t-test. For multiple comparisons of quantitative data, one-way analysis of variance was used. If the data fulfilled the criteria of homogeneity of variance, Fisher's least significant difference method was applied; otherwise, Tamhane's method was applied. $\mathrm{P}<0.05$ was considered to indicate a statistically significant difference.

\section{Results}

EA treatment improves neurological deficits following cerebral ischemia injury. The effect of EA on neurological 
A

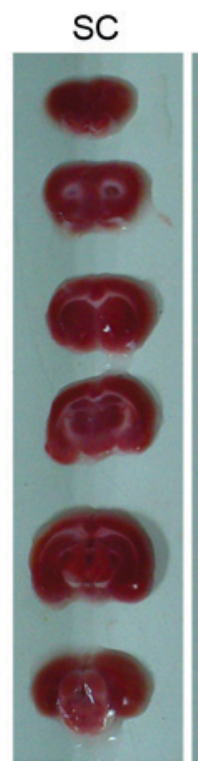

IC

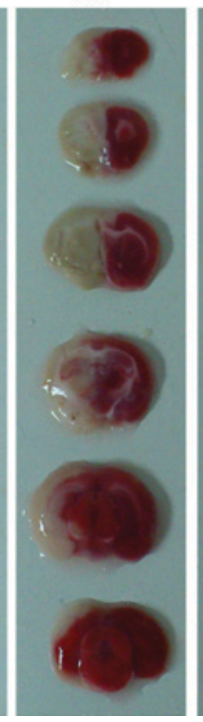

EA

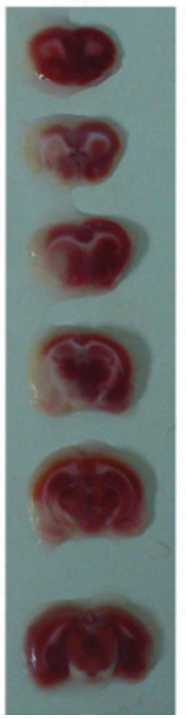

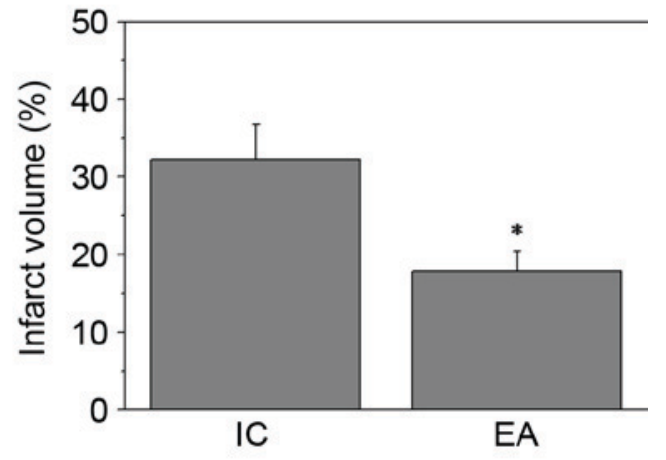

Figure 1. Effect of EA on infarct volumes. (A) 2,3,5-Triphenyltetrazolium chloride staining indicating cerebral infarct volumes of SC, IC and EA groups and (B) bar graph showing the percentage of total brain volume in each group. Values are expressed as the mean \pm standard deviation ( $\mathrm{n}=3$ ). ${ }^{*} \mathrm{P}<0.05$ vs. IC group. SC, sham-operated control; IC, ischemic control; EA, electroacupuncture.

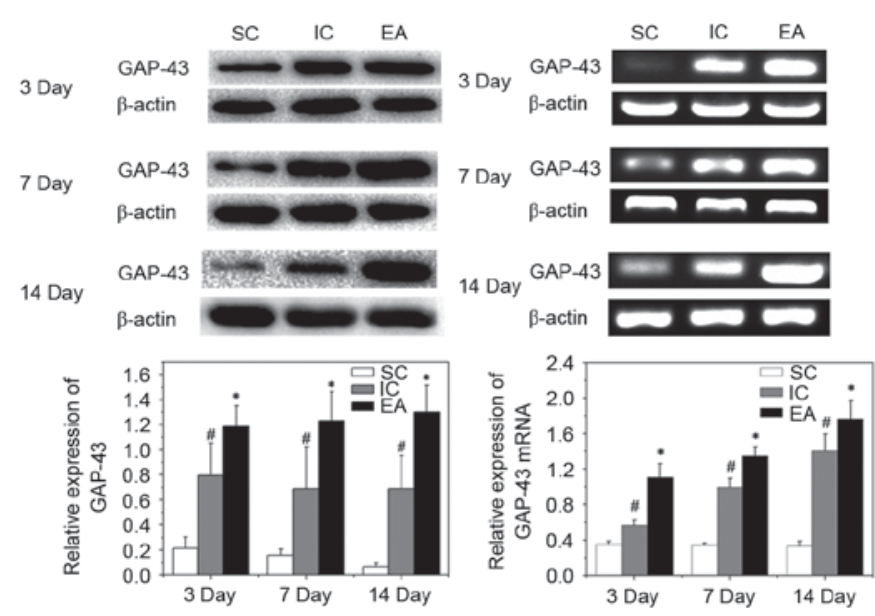

Figure 2. Effect of EA on GAP-43 in rats with cerebral ischemia injury. The protein expression and mRNA levels of GAP-43 in ischemic cerebral tissues on day 3,7 and 14 were determined by western blot and reverse-transcription quantitative polymerase chain reaction analyses. Values are expressed as the mean \pm standard deviation from 6 independent experiments. ${ }^{~} \mathrm{P}<0.05$ vs. SC group; " $\mathrm{P}<0.05$ vs. IC group. SC, sham-operated control; IC, ischemic control; EA, electroacupuncture; GAP, growth-associated protein.

function following cerebral ischemic injury was examined by neurological deficit scoring. As presented in Table I, there was no manifestation of neurological deficits observed in the SC group at each time-point, however rats in the IC and EA groups exhibited marked neurological deficits. The results indicated that successful cerebral ischemic models were established and rats in the SC group did not exhibit any indication of cerebral injury. There were no significant differences between the neurological deficit scores of the IC and EA groups at 2 and $24 \mathrm{~h}$ following cerebral ischemic injury $(\mathrm{P}>0.05)$. However, the scores in the EA group were significantly lower than those in the IC group at 3, 7 and 14 days $(\mathrm{P}<0.01$ or $\mathrm{P}<0.05$; Table $\mathrm{I})$ and gradually decreased

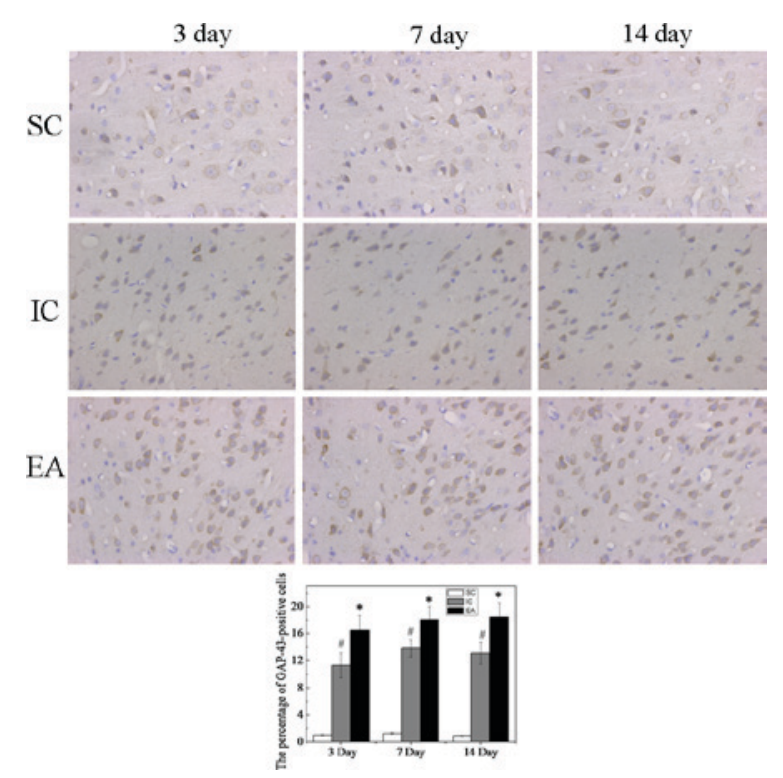

Figure 3. Effect of EA on GAP-43 in cerebral ischemic injured rats. Cerebral tissues from each group at different time-points were processed for an immunohistochemical assay. GAP-43-positive cells exhibited brown staining in the ischemic cerebral area (magnification, $\mathrm{x} 400$ ) at each time-point. Images are representative of six individual rats in each group. ${ }^{\#} \mathrm{P}<0.05$ vs. $\mathrm{SC}$ group; "P<0.05 vs. IC group. SC, sham-operated control; IC, ischemic control; EA, electroacupuncture; GAP, growth-associated protein.

over time. These results suggest that EA effectively improved the neurological dysfunction caused by cerebral ischemia and promoted the functional recovery of nerves.

EA reduces cerebral infarct volumes. To further investigate the therapeutic efficacy of EA treatment against cerebral ischemia injury, cerebral infarct volumes were evaluated using TTC staining. Sections of the SC group were stained red, whereas the unstained infarct brain area was visible on the left side in the IC and EA groups (Fig. 1A). Furthermore, EA treatment at 

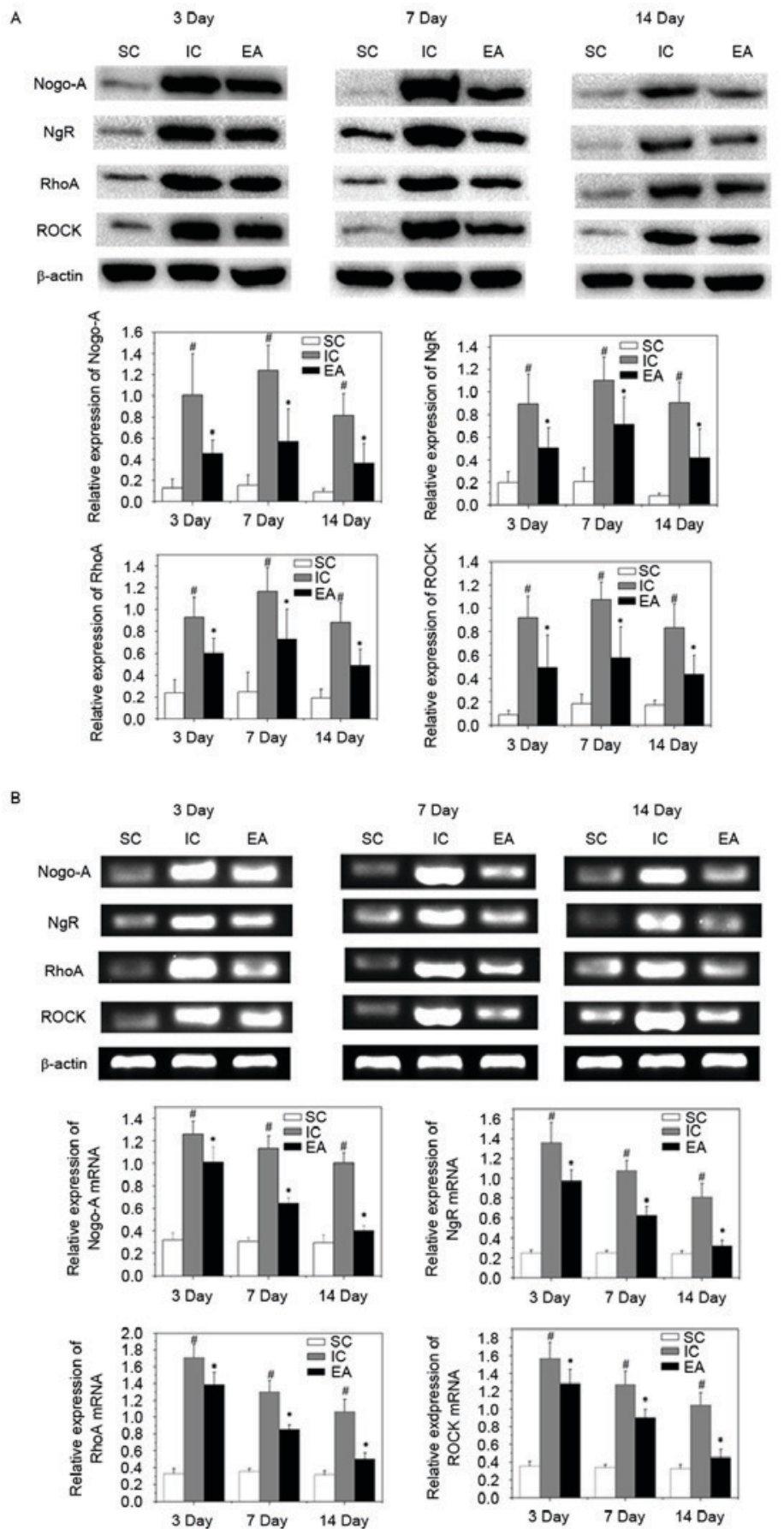

Figure 4. Effect of EA on the Nogo-A/NgR/RhoA/ROCK signaling pathway in rats with cerebral ischemic injury. (A) The protein expression of Nogo-A/NgR/RhoA/ROCKinischemiccerebraltissuesonday3,7 and 14 wasdeterminedbywesternblotanalysis.(B)ThemRNAlevelsofNogo-A/NgR/RhoA/ROCK in ischemic cerebral tissues on day 3,7 and 14 were determined by reverse-transcription quantitative polymerase chain reaction. Values are expressed as the mean \pm standard deviation from 6 independent experiments. " $\mathrm{P}<0.05$ vs. SC group; ${ }^{*} \mathrm{P}<0.05$ vs. IC group. SC, sham-operated control; IC, ischemic control; EA, electroacupuncture; NgR, Nogo receptor; ROCK, Rho-associated protein kinase.

the Quchi and Zusanli acupoints significantly reduced cerebral infarct volumes $(\mathrm{P}<0.05$; Fig. 1B).

Impact of EA treatment on axonal regeneration. Axonal regeneration can be identified by the elevated expression of GAP-43. GAP-43 is a major protein of axonal growth cones and has been implicated in the mechanism of axonal regeneration (21). To identify whether the expression of various axonal and myelin markers in the peri-infarct cortex were affected by EA treatment, western blotting, RT-qPCR and
IHC analyses of GAP-43 in the peri-infarct cortex were performed. Compared with the SC group, expression of GAP-43 mRNA and protein increased following cerebral ischemia in the IC and EA groups $(\mathrm{P}<0.05)$. Furthermore, EA treatment significantly enhanced the expression of GAP-43 protein and mRNA on days 3,7 and 14 post-ischemia, compared with the IC group ( $\mathrm{P}<0.05$; Fig. 2). Immunohistochemical staining indicated that only few GAP-43-positive cells were detected in the SC group, whereas the number of GAP-43-positive cells was increased in the IC and EA groups 
A
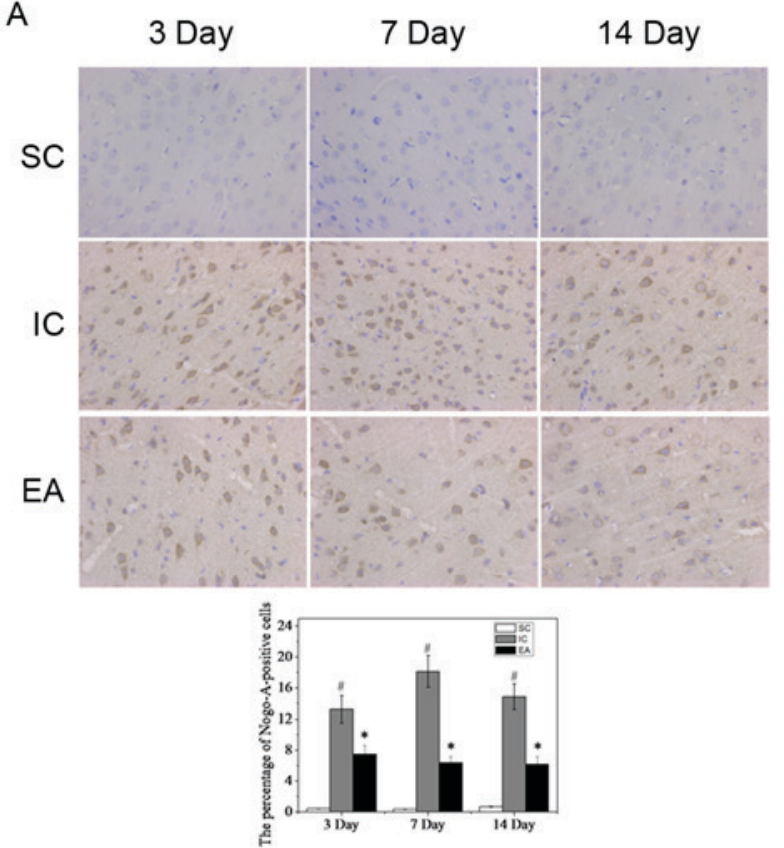

B
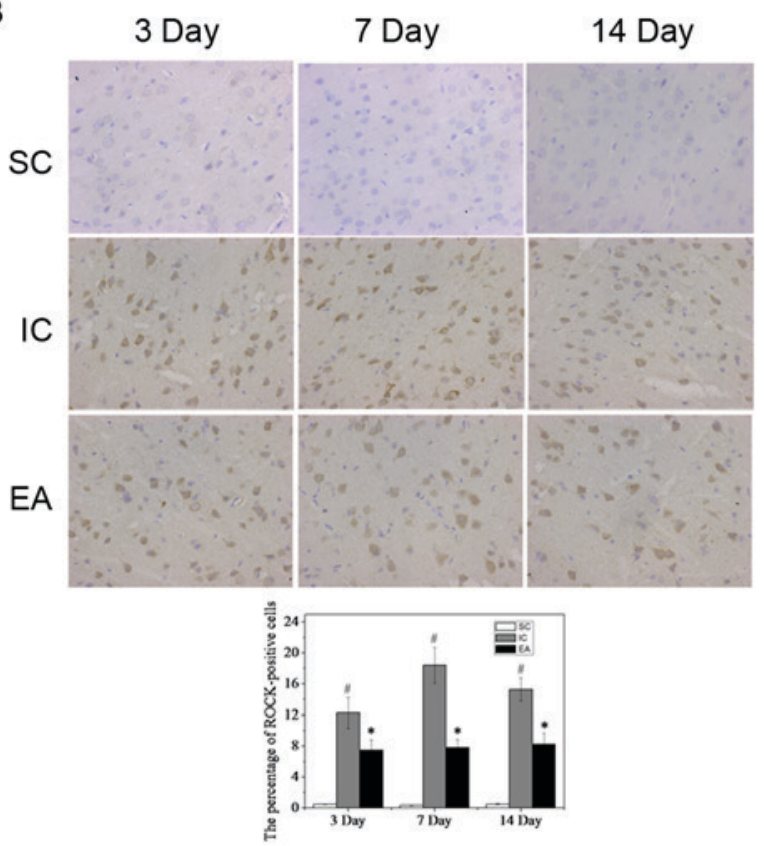

Figure 5. Effect of EA on Nogo-A and ROCK in cerebral ischemic injured rats. (A) Cerebral tissues from each group at different time point were processed for an immunohistochemical assay. Nogo-A-positive cells exhibited brown staining in the ischemic cerebral area (magnification, $\mathrm{x} 400)$ at each time-point. (B) ROCK expression at different time-points. Images are representative of six individual rats in each group. ${ }^{\#} \mathrm{P}<0.05$ vs. $\mathrm{SC}$ group; ${ }^{*} \mathrm{P}<0.05 \mathrm{vs}$. IC group. $\mathrm{SC}$, sham-operated control; IC, ischemic control; EA, electroacupuncture; ROCK, Rho-associated protein kinase.

(Fig. 3). The percentage of GAP-43-positive cells in the SC group at day 3 was $1.04 \pm 0.07 \%$, while those in the IC and EA groups were significantly higher $(\mathrm{P}<0.05)$, at $11.32 \pm 1.85$ and $16.51 \pm 2.17 \%$, respectively. This was also the case at days 7 and $14(\mathrm{P}<0.05)$. Furthermore, compared with the IC group, the number of GAP-43-positive cells was significantly increased in the EA group at days 3, 7 and $14(\mathrm{P}<0.05$, Fig. 3$)$.

Effect of EA treatment on activation of Nogo-A/NgR/RhoA/ROCK signaling. To investigate the mechanism of axonal regeneration with EA treatment, the expression of the vital target genes of the Nogo-A signaling pathway, Nogo-A, NgR, RhoA and ROCK, was investigated. The expression of Nogo-A, NgR, RhoA and ROCK mRNA and protein was significantly increased in the IC group compared with the SC group at on days 3, 7 and 14 (all $\mathrm{P}<0.05$ ). However, expression of Nogo-A, NgR, RhoA and ROCK mRNA and protein were significantly inhibited compared with the IC group, following EA treatment (all $\mathrm{P}<0.05$; Fig. 4). To confirm these results, Nogo-A and ROCK were detected in the peri-infarct cortex tissue by immunohistochemistry. As expected, few Nogo-A- and ROCK-positive cells were observed in the SC group (Fig. 5). By contrast, in the IC group, Nogo-A and ROCK expression significantly increased on day $3(13.24 \pm 1.74$ and $12.27 \pm 1.98 \%$, respectively; $\mathrm{P}<0.05)$, peaked on day 7 (18.13 \pm 2.06 and $18.41 \pm 2.31 \%$, respectively; $\mathrm{P}<0.05$ ), and then decreased slightly but was still maintained at a high level on day 14 (14.84 \pm 1.67 and $15.27 \pm 1.50 \%$, respectively; $\mathrm{P}<0.05$; Fig. 5). However, compared with the IC group, Nogo-A and ROCK expression significantly decreased in the EA group $(\mathrm{P}<0.05$; Fig. 5), suggesting that EA treatment weakened the inhibition of axonal growth following cerebral ischemia.

\section{Discussion}

Ischemic stroke has complex pathogenic mechanisms and its consequences are devastating. Various studies have identified that pathological alterations in neuronal cells within the gray matter occur following stroke. However, the importance of white matter integrity in long-term recovery from these conditions has also been highlighted (22-24). Brain white matter injury largely includes the injury of myelin-ensheathed axons, which, due to limited capacity of the adult brain for axonal regeneration, is strongly associated with long-term deficits in neurological function following stroke (25).

The molecular signaling in the brain following cerebral infarction is complex and includes inhibitors that reduce axonal regeneration and neurotrophic factors that promote synaptic regeneration. Nogo-A, as the pivotal myelin-associated axonal growth inhibitory protein, is a major impediment to axonal regeneration following ischemic stroke (26). Previous studies have shown that downregulation or inhibition of the Nogo-A signaling pathway promoted axonal regeneration and functional recovery of stroke (27-29). Nogo-A, via the $\mathrm{NgR}$ complex, activates RhoA and ROCK, ultimately resulting in the inhibition of axonal regeneration and growth cone collapse (15). Axonal regeneration is identified by the elevated expression of GAP-43. GAP-43 is a major protein of axonal growth cones and has been implicated in mechanisms of axonal regeneration (21). The present study demonstrated that EA treatment reduces the mitigating effect of cerebral ischemia on axonal growth inhibitors by downregulating the expression of Nogo-A/NgR/RhoA/ROCK signaling and upregulating the expression of Gap-43.

EA treatment, engrafted electric stimulation, is accepted as a complementary and alternative intervention for stroke as 
well as in post-stroke rehabilitation (30). A number of studies have demonstrated that EA treatment exerts a neuroprotective effect following cerebral ischemia stroke (31-33). It is thought that the neuroprotective mechanisms of EA include the promotion of neural cell proliferation (34), enhancement of endogenous neurogenesis (35), inhibition of neuronal apoptosis (36) and reduction of the inflammatory response (37).

In the present study, days 3, 7 and 14 after cerebral ischemia were selected as the time-points of observation, as they cover the key events of anti-apoptotic and neuroprotective mechanisms following cerebral ischemia: Day 3 lies within the trigger and onset period with minimal pathological changes and minimal edema; at day 7 , the progression of injury is maintained with prominent fiber degeneration and endoneurial edema; and from day 14, the period of regeneration is maintained with abundant small regenerating fiber clusters and minimal edema. In the IC group, significantly higher expression of the Nogo-A signaling genes was observed on day 3 with a peak on day 7 and a slight decrease on day 14, whereas this tendency was not observed in the EA group. Possible explanations may include the time-dependent treatment effects of EA stimulation as well as spontaneous recovery. However, the present finding that the EA group exhibited decreased expression of the Nogo-A signaling pathway compared with the IC group suggested that EA treatment weakened the inhibition of axonal growth following cerebral ischemia.

In conclusion, the present study determined that EA weakened the inhibition of axonal regrowth through the downregulation of Nogo-A/NgR/Rho-A/ROCK signaling following focal cerebral ischemia stroke, thus potentially contributing to the recovery of nerve function. Therefore, it may serve a role in protecting the brain following stroke. To the best of our knowledge, the present study was the first to demonstrate that EA at the Zusanli (ST36) and Quchi (LI11) acupoints on the paralyzed limb provided a less inhibitory environment for axonal regrowth following ischemic stroke. Furthermore, these results suggested that EA may have marked effects on white matter plasticity and regulation of the microenvironment of axonal regeneration around the infarction area. The present study also provided a molecular mechanism for the therapeutic effects of EA to promote stroke recovery.

\section{Acknowledgements}

The present study was financially supported by the Natural Science Foundation of Fujian Province, China (grant no. 2014J01345) and Innovative Medicine Subject of Fujian Provincial Health and Family Planning Commission, China (grant no. 2016-CX-47). The authors would like to thank the Fujian Provincial Rehabilitation industrial institution, Fujian University of Traditional Chinese Medicine, China for assistance.

\section{References}

1. Berkowitz AL: Stroke and the noncommunicable diseases: A global burden in need of global advocacy. Neurology 84: 2183-2184, 2015.

2. Corbyn Z: Statistics: A growing global burden. Nature 510: S2-S3, 2014.
3. Lloyd-Jones D, Adams R, Carnethon M, De Simone G, Ferguson TB, Flegal K, Ford E, Furie K, Go A, Greenlund K, et al: heart disease and stroke statistics-2009 update: A report from the American heart association statistics committee and stroke statistics subcommittee. Circulation 119: e21-e181, 2009.

4. Merson TD and Bourne JA: Endogenous neurogenesis following ischaemic brain injury: Insights for therapeutic strategies. Int J Biochem Cell Biol 56: 4-19, 2014.

5. Go AS, Mozaffarian D, Roger VL, Benjamin EJ, Berry JD, Borden WB, Bravata DM, Dai S, Ford ES, Fox CS, et al: Heart disease and stroke statistics-2013 update: A report from the American heart association. Circulation 127: e6-e245, 2013.

6. Tan F, Wang X, Li HQ, Lu L, Li M, Li JH, Fang M, Meng D and Zheng GQ: A randomized controlled pilot study of the triple stimulation technique in the assessment of electroacupuncture for motor function recovery in patients with acute ischemic stroke. Evid Based Complement Alternat Med 2013: 431986, 2013.

7. Fang Z, Ning J, Xiong C and Shulin Y: Effects of electroacupuncture at head points on the function of cerebral motor areas in stroke patients: A pet study. Evid Based Complement Alternat Med 2012: 902413, 2012.

8. Tao J, Chen B, Gao Y, Yang S, Huang J, Jiang X, Wu Y, Peng J, Hong $\mathrm{Z}$ and Chen L: Electroacupuncture enhances hippocampal NSCs proliferation in cerebral ischemia-reperfusion injured rats via activation of notch signaling pathway. Int J Neurosci 124: 204-212, 2014.

9. Xie G, Yang S, Chen A, Lan L, Lin Z, Gao Y, Huang J, Lin J, Peng J, Tao J and Chen L: Electroacupuncture at Quchi and Zusanli treats cerebral ischemia-reperfusion injury through activation of ERK signaling. Exp Ther Med 5: 1593-1597, 2013.

10. Chen A, Lin Z, Lan L, Xie G, Huang J, Lin J, Peng J, Tao J and Chen L: Electroacupuncture at the Quchi and Zusanli acupoints exerts neuroprotective role in cerebral ischemia-reperfusion injured rats via activation of the PI3K/AKT pathway. Int J Mol Med 30: 791-796, 2012.

11. Hinman JD: The back and forth of axonal injury and repair after stroke. Curr Opin Nrueol 27: 615-623, 2014.

12. Benowitz LI and Carmichael ST: Promoting axonal rewiring to improve outcome after stroke. Neurobiol Dis 37: 259-266, 2010.

13. Schwab ME and Strittmatter SM: Nogo limits neural plasticity and recovery from injury. Curr Opin Neurobiol 27: 53-60, 2014.

14. Wälchli T, Pernet V, Weinmann O, Shiu JY, Guzik-Kornacka A, Decrey G, Yüksel D, Schneider H, Vogel J, Ingber DE, et al: Nogo-A is a negative regulator of CNS angiogenesis. Proc Natl Acad Sci USA 110: E1943-E1952, 2013.

15. Zagrebelsky $M$ and Korte $M$ : Maintaining stable memory engrams: New roles for Nogo-A in the CNS. Neuroscience 283: 17-25, 2014

16. Lindau NT, Bänninger BJ, Gullo M, Good NA, Bachmann LC, Starkey ML and Schwab ME: Rewiring of the corticospinal tract in the adult rat after unilateral stroke and anti-Nogo-A therapy. Brain 137: 739-756, 2014.

17. Tsai SY, Papadopoulos CM, Schwab ME and Kartje GL: Delayed anti-Nogo-a therapy improves function after chronic stroke in adult rats. Steoke 42: 186-190, 2011.

18. National Research Council (US) Committee for the Update of the Guide for the Care and Use of Laboratory Animals: Guide for the Care and Use of Laboratory Animals. National Academies Press (US), Washington (DC) pp 85-23, 1996.

19. Longa EZ, Weinstein PR, Carlson S and Cummins R: Reversible middle cerebral artery occlusion without craniectomy in rats. Steoke 20: 84-91, 1989.

20. Bederson JB, Pitts LH, Tsuji M, Nishimura MC, Davis RL and Bartkowski H: Rat middle cerebral artery occlusion: Evaluation of the model and development of a neurologic examination. Steoke 17: 472-476, 1986.

21. Benowitz LI and Routtenberg A: GAP-43: An intrinsic determinant of neuronal development and plasticity. Trends Neurosci 20: 84-91, 1997.

22. Rosenzweig S and Carmichael ST: The axon-glia unit in white matter stroke: Mechanisms of damage and recovery. Brain Res 1623: 123-134, 2015.

23. Suenaga J, Hu X, Pu H, Shi Y, Hassan SH, Xu M, Leak RK, Stetler RA, Gao Y and Chen J: White matter injury and microglia/macrophage polarization are strongly linked with age-related long-term deficits in neurological function after stroke. Exp Neurol 272: 109-119, 2015.

24. Matute C, Domercq M, Pérez-Samartin A and Ransom BR: Protecting white matter from stroke injury. Stroke 44: 1204-1211, 2013. 
25. Shi H, Hu X, Leak RK, Shi Y, An C, Suenaga J, Chen J and Gao Y: Demyelination as a rational therapeutic target for ischemic or traumatic brain injury. Exp Neurol 272: 17-25, 2015.

26. Kumar P and Moon LD: Therapeutics targeting Nogo-A hold promise for stroke restoration. CNS Neurol Disord Drug Targets 12: 200-208, 2013.

27. Li C, Wen H, Wang Q, Zhang C, Jiang L, Dou Z, Luo X and Zeng J: Exercise training inhibits the Nogo-A/NgR1/Rho-A signals in the cortical peri-infarct area in hypertensive stroke rats. Am J Phys Med Rehabil 94: 1083-1094, 2015.

28. Liu L, Zhu L, Zou Y, Liu W, Zhang X, Wei X, Hu B and Chen J: Panax notoginseng saponins promotes stroke recovery by influencing expression of Nogo-A, NgR and p75NGF, in vitro and in vivo. Biol Pharm Bull 37: 560-568, 2014.

29. Chen X, Wang N, Liu Y, Liu Y, Zhang T, Zhu L, Wang Y, Wu C and Yang J: Yonkenafil: A novel phosphodiesterase type 5 inhibitor induces neuronal network potentiation by a cGMP-dependent Nogo-R axis in acute experimental stroke. Exp Neurol 261: 267-277, 2014

30. Wu P, Mills E, Moher D and Seely D: Acupuncture in poststroke rehabilitation: A systematic review and meta-analysis of randomized trials. Stroke 41: e171-e179, 2010.

31. Zhao Y, Deng B, Li Y, Zhou L, Yang L, Gou X, Wang Q, Chen $\mathrm{G}, \mathrm{Xu} \mathrm{H}$ and $\mathrm{Xu} \mathrm{L}$ : Electroacupuncture pretreatment attenuates cerebral ischemic injury via notch pathway-mediated up-regulation of hypoxia inducible factor-1 $\alpha$ in rats. Cell Mol Neurobiol 35: 1093-1103, 2015.
32. Chen C, Zhang W, Lou BD, Pan J, Cao Y, Zhong F, Zhou WJ and Wu J: Effect of Electroacupuncture stimulation of acupoints of the Pericardium Meridian on serum NGF and Nogo-A contents and cerebral NGF and Nogo-A expression in cerebral ischemia rats. Zhen Ci Yan Jiu 40: 94-98, 2015 (In Chinese).

33. Cheng CY, Lin JG, Su SY, Tang NY, Kao ST and Hsieh CL: Electroacupuncture-like stimulation at Baihui and Dazhui acupoints exerts neuroprotective effects through activation of the brain-derived neurotrophic factor-mediated MEK1/2/ERK1/2/p90RSK/bad signaling pathway in mild transient focal cerebral ischemia in rats. BMC Complement Altern Med 14: 92, 2014.

34. Huang J, Ye X, You Y, Liu W, Gao Y, Yang S, Peng J, Hong Z, Tao J and Chen L: Electroacupuncture promotes neural cell proliferation in vivo through activation of the ERK1/2 signaling pathway. Int J Mol Med 33: 1547-1553, 2014

35. Kim YR, Kim HN, Ahn SM, Choi YH, Shin HK and Choi BT: Electroacupuncture promotes post-stroke functional recovery via enhancing endogenous neurogenesis in mouse focal cerebral ischemia. PLoS One 9: e90000, 2014.

36. Xue X, You Y, Tao J, Ye X, Huang J, Yang S, Lin Z, Hong Z, Peng J and Chen L: Electro-acupuncture at points of Zusanli and Quchi exerts anti-apoptotic effect through the modulation of PI3K/Akt signaling pathway. Neurosci Lett 558: 14-19, 2014.

37. Lan L, Tao J, Chen A, Xie G, Huang J, Lin J, Peng J and Chen L: Electroacupuncture exerts anti-inflammatory effects in cerebral ischemia-reperfusion injured rats via suppression of the TLR4/NF-kB pathway. Int J Mol Med 31: 75-80, 2013. 